\title{
RAPID AWARENESS AND TRANSMISSION OF SEVERE ACUTE RESPIRATORY SYNDROME IN HANOI FRENCH HOSPITAL, VIETNAM
}

\author{
HIROSHI NISHIURA, TADATOSHI KURATSUJI,* TRAN QUY, NGUYEN CHI PHI, VO VAN BAN, LE DANG HA, \\ HOANG THUY LONG, HIDEKI YANAI, NAOTO KEICHO, TERUO KIRIKAE, TAKEHIKO SASAZUKI, AND \\ ROY M. ANDERSON \\ The Research Institute of Tuberculosis, Japan Anti-Tuberculosis Association, Tokyo, Japan; Department of Infectious Disease \\ Epidemiology, Faculty of Medicine, Imperial College London, United Kingdom; Research Institute, International Medical Center of \\ Japan, Tokyo, Japan; Bach Mai Hospital, Hanoi, Vietnam; Hanoi-French Hospital, Hanoi, Vietnam; National Institute for Clinical \\ Research in Tropical Medicine, Hanoi, Vietnam; National Institute of Hygiene and Epidemiology, Hanoi, Vietnam
}

\begin{abstract}
A case-control study was conducted to examine the relationship between severe acute respiratory syndrome (SARS) and the time-dependent precautionary behaviors taken during an outbreak of SARS in Hanoi French Hospital $(\mathrm{HFH})$, Vietnam. Masks (odds ratio $[\mathrm{OR}]=0.3 ; 95 \%$ confidence interval $[\mathrm{CI}]: 0.1,0.7)$ and gowns $(\mathrm{OR}=0.2$; 95\% CI: $0.0,0.8)$ appeared to prevent SARS transmission. The proportion of doctors and nurses who undertook each measure significantly improved $\left(\chi^{2}=9.8551, P=0.043\right)$ after the onset of secondary cases. The impact of individual behaviors on an outbreak was investigated through mathematical approaches. The reproduction number decreased from 4.1 to 0.7 after notification. The basic reproduction number was estimated, and the use of masks alone was shown to be insufficient in containing an epidemic. Intuitive results obtained by means of stochastic individual-based simulations showed that rapid improvements in behavior and isolation would increase the probability of extinction.
\end{abstract}

\section{INTRODUCTION}

Notwithstanding the announcement of containment by the World Health Organization (WHO) in 2003, ${ }^{1}$ severe acute respiratory syndrome (SARS) has remained a matter of concern worldwide, and it is not surprising that several cases of SARS have reemerged, for example, in China in April 2004. ${ }^{2}$ Although the mode of transmission remains partially unclear, especially with regard to airborne transmission ${ }^{3}$ and superspreading events, ${ }^{4,5}$ it appears to occur predominantly by large droplets, direct contact with infectious material, or contact with fomites contaminated with infectious material., ${ }^{6,7}$ The most effective containment measures identified to date include the tracing of contacts, ${ }^{8}$ quarantine, ${ }^{9}$ triage and early case detection, ${ }^{10,11}$ and isolation. ${ }^{12}$ Further, because the close contact required for transmission easily occurs in hospital settings, ${ }^{13-15}$ nosocomial spread was determined as one of the major epidemiologic features of SARS. ${ }^{7,16,17}$ The elimination of hospital transmission through enhanced infection control practices is therefore a crucial control measure.

An early study in Hong Kong showed that the practice of droplet and contact precautions was adequate in most clinical settings in significantly reducing the risk of infection after exposure to patients with SARS, ${ }^{18}$ and if practiced by a high proportion of susceptible individuals, precautionary measures are expected to significantly reduce transmission. ${ }^{19}$ The adoption of routine preventive behaviors based on appropriate training and control among health care workers (HCWs), undertaken prior to the isolation of SARS patients, was shown to be one of the most crucial control measures. ${ }^{20-22}$

In this context, Vietnam is considered to have achieved the first highly successful containment of SARS during the early phase of the outbreak. ${ }^{23}$ One reason for this rapid containment is thought to be the prevention of infection leakage from hospitals back into the general community. ${ }^{24}$ A second is the successful discontinuation of the chain of nosocomial

\footnotetext{
* Address correspondence to Tadatoshi Kuratsuji, Research Institute, International Medical Center of Japan, Toyama 1-21-1, Shinjukuku, Tokyo, 162-8655, Japan. E-mail: kuratsuji@ri.imcj.go.jp
}

transmission several days after onset based on the radical control measures of the Ministry of Health, Vietnam. ${ }^{25} \mathrm{Al}-$ though several nosocomial transmissions were observed in Hanoi French Hospital (HFH) in the early days of the outbreak, ${ }^{26,27}$ none were identified in HFH or another local hospital in the latter phase. ${ }^{28}$ In both hospitals, staff instituted stringent precautions, strict isolations, and quarantines under the encouragement of Dr. Carlo Urbani (Dr. Urbani died of SARS before seeing the success of the containment). ${ }^{29} \mathrm{We}$ therefore consider that a comprehensive understanding of the successful containment measures adopted by HFH and their theoretical underpinnings are crucial to the success of control strategies for any future recurrence. Here, we use a casecontrol study design to time-dependently examine the relationship between SARS and the precautionary behaviors undertaken by those exposed in HFH. We then use mathematical approaches to develop intuitive analyses of the impact of individual behaviors on the control of a SARS epidemic.

\section{MATERIALS AND METHODS}

Case-control study. HFH is a 56-bed secondary care hospital. After the admission of an index case on February 26, 2003, 38 cases in total were confirmed to have symptomatic SARS infection. The occurrence of newly diagnosed SARS cases due to local transmission continued until April 7, 2003, 3 weeks before the date when the Vietnamese government and WHO declared the outbreak successfully contained (April 28, 2003) (Table 1). The duration of the HFH outbreak was analyzed by separating it into three phases: Stage 1, February 26-March 4, from admission of the index case to the onset of secondary cases; Stage 2, March 5-March 10, from the suspicion of nosocomial spread to closure of the hospital; and Stage 3, from March 11 on, from strict isolation to local eradication.

A case-control study of 29 of the 38 laboratory-confirmed SARS cases and 98 controls was performed in HFH. The case group included 22 of $28(78.6 \%)$ individuals admitted and retained in HFH and 7 of $10(70.0 \%)$ individuals transferred to another hospital after first being admitted to HFH (total $N=29$ ). The reasons for nonparticipation were death due to 
TABLE 1

Chronology of the outbreak of SARS in Hanoi French Hospital $(\mathrm{HFH})$, Vietnam

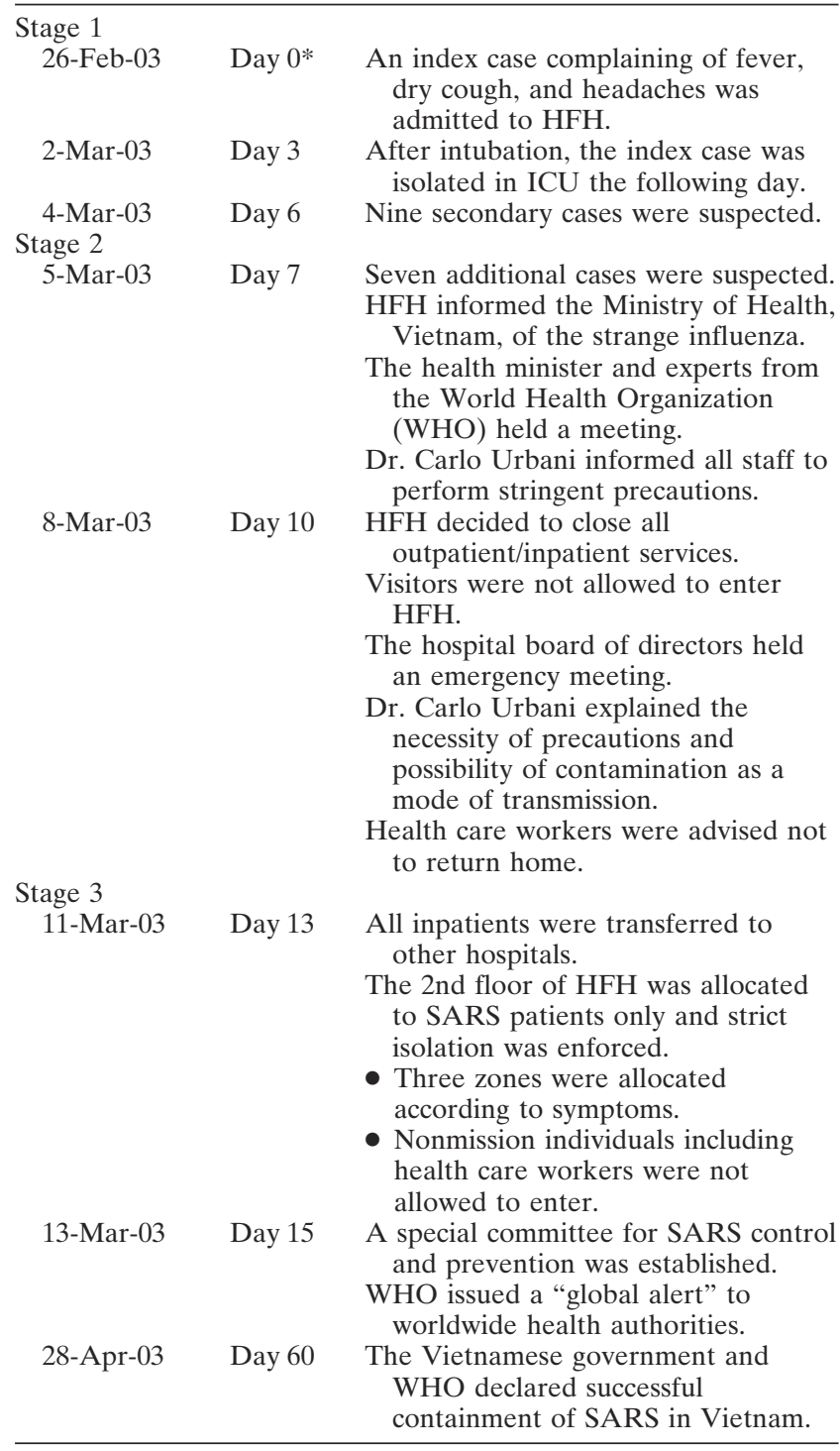

Day, days after onset of the outbreak. SARS, severe acute respiratory syndrome; ICU, intensive care unit.

SARS and/or respiratory failure $(N=5,13.2 \%)$, refusal to take part $(N=1,2.6 \%)$, or relocation $(N=3,7.9 \%)$. The case group included $28 \mathrm{HFH}$ employees (3 doctors, 13 nurses and nursing assistants, 10 radiologists and other co-medical workers, and 2 receptionist and administrative staff) and 1 relative of a patient. A further 23 Vietnamese patients who were directly admitted to another hospital were excluded because the detailed source of infection was unknown, although several cases were thought to have been infected in HFH. Detailed descriptions of the laboratory diagnoses were given previously. ${ }^{28}$ They were confirmed through serological studies using an indirect enzyme-linked immunosorbent assay (ELISA) (Kirikae T, et al., unpublished data).

Controls were nominated based on employment in $\mathrm{HFH}$ and exposure among patients' relatives through HFH. The selection criteria included i) Vietnamese individuals more than 20 years old, ii) those who provided written informed consent based on explanation of our methods and purposes, and iii) those thought to have had contact with confirmed cases inside the hospital based on contact investigations. In total, 98 individuals were included as controls; most were HFH employees (13 doctors, 20 nurses and nursing assistants, 13 radiologists and other co-medical workers, and 11 receptionists and administrative staffs) or relatives of patients $(N=41)$. Although we investigated certain known contacts for inclusion as controls, namely individuals who took care of cases or entered cases' room, those who might have had trivial contact, such as possible exposure outside the hospital during, for example, transportation of SARS cases or in the casualty reception room, were not followed and included. The number of hospital employees investigated represented approximately $55.9 \%$ of the total employees used during the outbreak.

All participants were surveyed with regards to their use of personal protective equipment (PPE) and hygiene habits when in contact with patients with SARS; that is, the use of masks, gloves, and gowns, and the practice of hand washing, which were specifically recommended as droplet and contact precautions. In this paper, masks denote surgical masks; N95 masks were not available in the early stage of the outbreak in Vietnam. Individual behaviors were investigated mainly in two separate phases according to time-dependency (in Stage 1 and after entering Stage 2; i.e., Stages 2 and 3) (Table 1) to clarify any behavioral changes that occurred. Standardized questionnaires requiring one of two possible answers for each precaution ("performed" or "not performed") were given to each subject, and all responses were collected. Answers of "sometimes" or "seldom" were defined as "not performed" due to imperfect efficacy. In addition, the frequency of contact with infected individuals was investigated to represent the number of exposures per day. An exposure result of "many times" was recorded for those who had close contact with SARS patients, that is, those who cared for or lived with SARS patients, and those likely to have come into direct contact with the respiratory secretions or body fluids of SARS patients, for example, during close conversation (within 3 feet). ${ }^{30}$ After completing the initial primary survey, an identical confirmation survey was performed to confirm the validity of the answers. These surveys were conducted along with other epidemiologic studies (Nishiyama A, et al., unpublished data) until mid-March 2004, almost 1 year after onset of the epidemic. No blood test results showing possible asymptomatic infections were available during the survey period. The participants were informed of how the information would be used and assured of the confidentiality of their responses. The purpose of the study was explained in Vietnamese, and written informed consent was obtained.

Statistical analyses were performed as follows. First, univariate associations between precautionary behaviors and infection were investigated in two separate stages (Stage 1 or Stages 2 and 3). Comparisons between groups were made using the $\chi^{2}$ or Fisher's exact test for univariate analysis. Multivariate logistic regression was done in Stage 1 using forward stepwise selection (Waldesian) to determine the most significant variable associated with protection among those studied. Significant steps were taken to minimize recall bias with Stages 2 and 3 data. Analysis was restricted to those who had probable contact in these stages. It was further restricted to those cases developing symptoms whose incubation period 
was within the greater than $95 \%$ confidence interval $(95 \%$ CI) of having occurred after the beginning of Stage 2; and finally to medical doctors and nurses only, for both cases and controls. Second, univariate associations between sociodemographic variables (sex, age, and occupation) and SARS were investigated, with age and occupation categorized into four different groups each. Third, interactions between the identified most significant protective behavior and other variables significantly associated in univariate analysis were investigated through the use of crosstabs statistics, in which the odds of being infected were stratified according to a comparison of variables, and interactions were sought through the different odds ratio in each strata. Finally, multiple logistic regression analysis was used to determine the protective effect and eliminate confounding variables. As described in the next section, all variables significantly associated in univariate analyses, as well as sociodemographic variables, were selected and entered together in the final model. All data were entered into Microsoft Excel 2000 (Microsoft Co., Redmond, WA), and the statistical data were analyzed using the statistical software "R" (R Development Core Team, Vienna). ${ }^{31}$

Mathematical methods. The predictive effects of the behavioral changes were simulated using an individual-based stochastic model. For ease of understanding, a compartmental model, a type of SEIR (susceptible $[S]$, exposed $[E]$, infected $[I]$, and recovered/removed $[R]$ ) model, which considered the process of transmission according to the protective behaviors taken against infectious contact among susceptible individuals, was applied. Instead of assuming "exposed (latent)" and "infectious" periods, $E$ and $I$ were defined as "incubation" and "symptomatic" periods, respectively, as the infectious period of SARS has not been fully clarified. Although SEIR models are usually deterministic and use mean estimations as model parameters, even with regard to SARS, ${ }^{12,32}$ stochastic simulations were performed in this study because of the need to consider the stochasticity of each protective behavior, and also because of the small sample population size. The infectious lifetime of each individual was presented as an absorbing Markov chain. The simulations start with an individual index case (Day 0) in a population of 300 in which all individuals are susceptible.

Of the total 127 subjects studied (29 cases and 98 controls), $62.2 \%(N=79)$ were considered to have had casual contact and $37.8 \%(N=48)$ to have had close contact with SARS patients. The number of casual contacts $\left(\kappa_{1}\right)$ was directly obtained $\left(=0.7 \pm 0.2\left[\mathrm{day}^{-1}\right]\right)$, while the mean of close contacts $\left(\kappa_{2}=0.4\left[\mathrm{day}^{-1}\right]\right)$ was determined with the following equation:

$$
\kappa_{2}=\kappa_{1} \ln \left(\mathrm{OR}_{\text {closed }}\right)
$$

where $\mathrm{OR}_{\text {closed }}(=2.5 ; 95 \% \mathrm{CI}$ : $1.1-5.9)$ denotes the odds ratio (OR) of getting infected as a result of close contact. In other words, to quantify close contact, we assumed that the frequency of infection is mainly determined by the frequency of contact, so that the ratio of the frequency of close to casual contact becomes proportional to the logarithm of the OR of transmission. The protective effect of precautionary behavior was approximated by:

$$
\beta=1-\mathrm{RR}=1-\frac{a(c+d)}{c(a+b)} \approx 1-\frac{a d}{b c} \approx 1-\mathrm{OR}
$$

where $\mathrm{RR}$ and $\mathrm{OR}$ denote the relative risk and odds ratio, respectively, of becoming infected while performing a protective behavior (with precaution $=$ with exposure). Here, $a$ is the number of exposed ill people; $b$, the number of exposed healthy people; $c$, the number of unexposed ill people; and $d$, the number of unexposed healthy people. If the outcome (i.e., disease investigated) is a rare event, that is, if $a$ and $c$ are very small compared with $b$ and $d$, respectively, $(a+b)$ and $(c+d)$, respectively, would be closely similar to $b$ and $d$ alone. In this case, OR would approximate RR.

The lengths of the incubation and symptomatic periods were both assumed to be independently and identically distributed random variables with a probability density function of $\gamma$ distribution, the mean and variance of which were defined as 3.8 [days] and 8.3 [days $^{2}$ ], and 16.2 [days] and 7.9 [days ${ }^{2}$ ], respectively. ${ }^{24,33}$ These distributions were applied to difference equations (as a discrete time model) by discretizing the probability density functions by day (for a detailed description of the simulation algorithm, see the Appendix).

The first simulation scenario hypothetically investigated the unchanged coverage and mean protective effects of a behavioral measure throughout the epidemic. Primary information on protective behaviors was obtained from our Stage 1 survey. Estimates for the extent of a protective effect, the associated causative behavior of which was found in forward stepwise logistic regression to be the most significantly associated with protection (as described above), were obtained through the use of further multivariate logistic regression analysis. This analysis incorporated all variables significantly associated with SARS on univariate analysis (i.e., other precautionary behavior, gender, age and occupation). To investigate the impact of the coverage of a protective measure on the trajectory of an outbreak, sensitivity of the cumulative number of SARS cases at Day 30 to the coverage of masks was investigated in the mean field equation. In the second scenario, it was assumed that coverage improved dramatically after entering Stage 2 (Day 7) due to an awareness of transmission. Further, in Stage 3 (Day 13), the hospital implemented not only stringent precautions but also strict isolations. To understand the trajectory of transmission in detail, the number of incubating as well as symptomatic individuals was investigated. As was in fact seen during Stage 3 of the outbreak, it was also assumed that all cases who became symptomatic were immediately isolated and that nobody except a limited number of healthcare workers were permitted to have contact with them. Because the greatest uncertainty applies to the time taken to increase coverage of a protective measure and to implement strict isolations, sensitivity analyses comparing the cumulative number of SARS cases up to Day 30 were performed with the time to change both protective measures set simultaneously on the same day. Finally, the basic reproduction number was estimated using the (effective) reproduction number obtained in Stage 1 (see Appendix).

\section{RESULTS}

Table 2 shows the univariate association between the precautionary behaviors taken (SARS and non-SARS [control] cases) in Stage 1 and SARS. The use of masks $(P=0.011)$ and gowns $(P=0.012)$ appeared to prevent infection, whereas handwashing and the use of gloves were less likely to provide protection. Only two subjects who performed all pro- 
TABLE 2

Precautionary measures taken by all participants in Stage 1

\begin{tabular}{lcccc}
\hline & $\begin{array}{c}\text { SARS cases } \\
(N=25)\end{array}$ & $\begin{array}{c}\text { Non-SARS } \\
(N=90)\end{array}$ & $P$ value* & $\begin{array}{c}\text { Odds ratio } \dagger \\
(95 \% \text { CI })\end{array}$ \\
\hline All measures & 2 & 44 & 0.059 & $0.2(0.0-1.0)$ \\
Handwashing before $\S$ & 12 & 51 & 0.937 & $1.0(0.4-2.3)$ \\
Handwashing afterप & 15 & 56 & 0.766 & $1.1(0.5-2.8)$ \\
Masks & 8 & 35 & 0.011 & $0.3(0.1-0.7)$ \\
Gloves & 8 & 30 & 0.643 & $0.7(0.3-1.9)$ \\
Gowns & 2 & 25 & 0.012 & $0.2(0.0-0.8)$ \\
\hline
\end{tabular}

* Two-tailed.

Odds ratio of being infected while taking specific precautions.

$\div 95 \%$ CI: $95 \%$ confidence interval.

$\S$ Hands washed before having contact with a patient

II Hands washed after having contact with a patient.

"Only those who always used a mask.

tective measures developed symptomatic infections $(P=$ $0.059)$. Forward stepwise logistic regression of the five protective measures $(0.05$ for entry and 0.10 for removal probability) showed that only the use of masks was significant in the final model (OR, 0.29, 95\% CI; 0.11-0.73, $P=0.009)$. In Stages 2 and 3 , the use of masks $(P=0.001)$ and gowns $(P=$ $0.010)$ was significantly associated with non-infection among doctors and nurses still not infected after Stage 1 (Table 3). Most performed all the personal protective measures recommended, and only one individual who wore masks was infected. The comparative results of the behaviors of all participants at Stage 1 and after entering Stage 2 are shown in Figure 1a. The proportions of individuals who performed the investigated protective behaviors increased after entering Stage 2. However, these behavioral changes were not significantly different between the two phases $(P=0.960)$. The behaviors performed by the doctors and nurses $(N=48$; Figure 1b) who had the closest contact with the SARS patients drastically and significantly improved after entering Stage $2\left(\chi^{2}=9.855, P=0.043\right)$.

The univariate associations between socio-demographic variables and SARS throughout the epidemic are shown in Table 4. Females were more likely to become infected than males $(P=0.011)$, and a significant association of SARS with nurses $(P=0.008)$ was observed. In HFH, infection was frequent in the $40-49$ age strata $(P=0.015)$. Among all study subject, relatives of patients $(P<0.001)$ appeared to be the least frequently infected. Table 5 shows the interaction between the use of masks and other significantly associated variables in univariate analyses. Even though we saw no signifi-

TABLE 3

Precautionary measures taken by health care workers in Stages 2 and 3

\begin{tabular}{|c|c|c|c|c|}
\hline & $\begin{array}{l}\text { SARS cases } \\
(N=4)\end{array}$ & $\begin{array}{l}\text { Non-SARS } \\
(N=26)\end{array}$ & $\begin{array}{c}P \\
\text { value* }\end{array}$ & $\begin{array}{l}\text { Odds ratio } \dagger \\
(95 \% \text { Cl }) \ddagger\end{array}$ \\
\hline All measures & 1 & 25 & 0.001 & $<0.1(0.0-0.3)$ \\
\hline Handwashing before $\S$ & 4 & 25 & 1.000 & $\mathrm{NC}$ \\
\hline Handwashing after $\mathbb{I}$ & 4 & 25 & 1.000 & $\mathrm{NC}$ \\
\hline Masks" & 1 & 25 & 0.001 & $<0.1(0.0-0.3)$ \\
\hline Gloves & 4 & 25 & 1.000 & $\mathrm{NC}$ \\
\hline Gowns & 3 & 26 & 0.010 & $\mathrm{NC}$ \\
\hline
\end{tabular}

cant difference in the OR of using masks versus the use of gowns, females $(\mathrm{OR}=0.2)$ and nurses $(\mathrm{OR}=0.1)$ were more effectively protected by the use of masks than others in Stage 1. In Stages 2 and 3, the use of gowns showed overall reasonable $\mathrm{OR}(=0.2)$, whereas most other interactions could not be calculated due to the scarcity of cases.

Figure 2a shows the mean and corresponding 95\% CI of the trajectory (shown as prevalence) of an epidemic from 250 simulation runs which hypothetically assumed unchanged coverage as well as the protective effects of the precautionary measures observed in Stage 1. The precautionary measure in this simulation was based on a multivariate logistic regression which included all variables showing significant associations in univariate analyses, and focused on the impact of the use of masks, given the identification of this behavior as the most important protective measure $(\beta=0.6$ obtained from $\mathrm{OR}=$ $0.4, P=0.020)$. The coverage of masks was obtained as $52.0 \%$ from Table 2 . If an outbreak was simply allowed to continue growing under these conditions, the results showed that approximately 50 to 90 symptomatic cases would occur by Day 30. The reproduction number $(R)$ was estimated as 4.1 (95\% CI; 1.9-6.4), and from this estimate the basic reproduction number was estimated as 6.0. Sensitivity of the cumulative number of cases to the coverage of masks, in the mean field, is shown in Figure 2b. Certain reduction in the cumulative number of cases was observed with significant improvements in coverage.

Figures $2 \mathrm{c}$ and $2 \mathrm{~d}$ shows the outbreak trajectory of 250 simulations assuming improved coverage (from 52.0 to $81.5 \%$ ) among susceptible individuals on Day 8 and restriction of contact with symptomatic individuals to health care workers on Day 13. The protective effect obtained from multivariage regression was $0.9(\mathrm{OR}=0.1, P=0.955)$. The reproduction number in Stage 2 was estimated as $0.7(95 \%$ CI; 0.0-2.3). The number of incubating individuals began to show a decreasing trend after these events (Figure 2c), followed by a declining trend in the number of symptomatic cases (Figure 2d). Most of the simulated outbreaks eventually declined to extinction before Day 120. The sensitivity of the final size of an epidemic, evaluated through observations of the cumulative numbers of cases, to the timing of drastic changes in protective behaviors accompanied by strict isolation is shown in Figure 2e. When the stochastic effects are taken into account together with the effects of single precautionary measures and isolation, the rapid implementation of combined measures reduces the number of transmissions and increases the probability of extinction.

\section{DISCUSSION}

The findings of this case-control study indicate that the use of masks was significantly associated with the prevention of SARS transmission and that precautions against droplet contamination and contact were adequate in preventing transmission; this implies mainly to in-hospitals. The results are roughly consistent with those of previous reports. ${ }^{18,20,22} \mathrm{Al}$ though a number of exceptions were seen with regard to protective effects during patient intubation, during which transmission to staff occurred even when droplet and contact precautions were taken, ${ }^{7,34}$ one of the most important lessons from the SARS outbreak is the need to enhance infection control programs in hospitals. ${ }^{13,35}$ Even though the use of 
a)

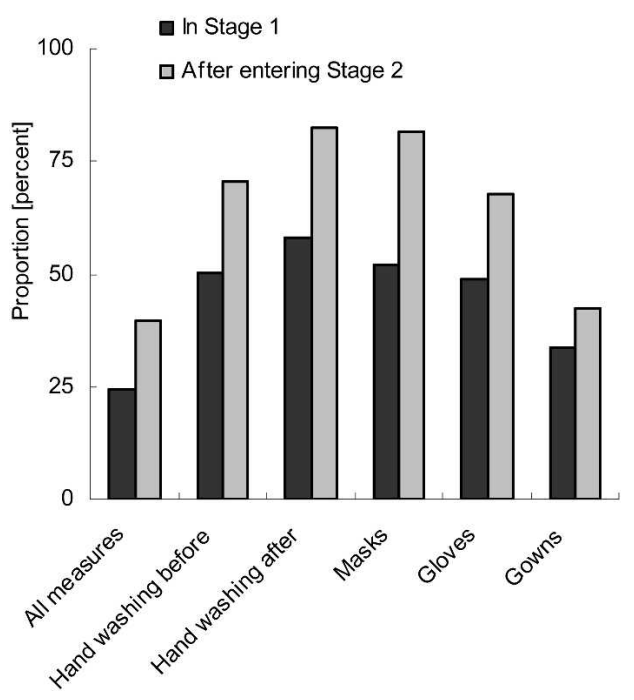

b)

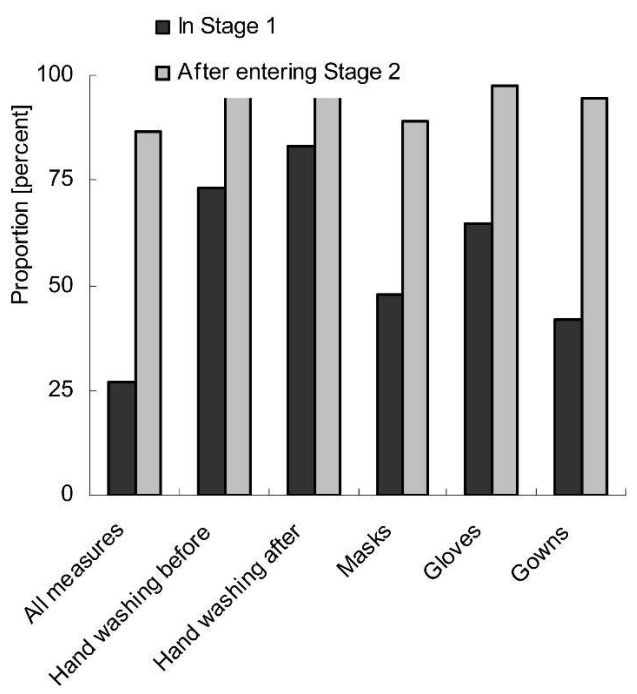

FIGURE 1. Protective behavioral changes defined by stage. a, Proportion of participants (SARS and non-SARS [control] cases) who performed each precautionary measure in Stage $1(N=127)$ and after entering Stage $2(N=108)$. Handwashing "before" and "after" denote before and after contact with a patient, respectively. $\mathbf{b}$, Proportion of health care workers who performed each precautionary measure in Stage $1(N=48)$ and after entering Stage $2(N=37)$.

masks was the most effective precautionary measure, masks alone together with the observed coverage did not reduce the reproduction number below unity $\left(R_{0}=6.0\right.$ and $R$ with the protective effects of masks $=4.1)$. Put simply, the use of masks alone was shown to be insufficient to contain the epidemic. Further, it was shown that the coverage of precautionary behaviors among the study subjects increased with the progression of the outbreak, and this was especially obvious among doctors and nurses. In HFH, remarkable changes occurred in the very early phase of the outbreak before detailed information about SARS was available. According to the stochastic simulations, an increased probability of extinction would be observed if the combined measures of precaution and isolation were rapidly implemented.

With regard to sociodemographic variables, females were more frequently infected than males. Given that transmission was most frequently observed among nurses, a plausible explanation for this finding would be occupational background. Although the 40-49 age group was frequently infected, we

TABLE 4

Univariate associations between age-class/occupational categories and SARS

\begin{tabular}{llccc}
\hline \multicolumn{1}{c}{ Category } & $N$ & $\begin{array}{c}P \\
\text { value* }\end{array}$ & $\begin{array}{c}\text { Odds ratio } \\
(95 \% \mathrm{CI}) \dagger\end{array}$ \\
\hline Sex & Male & 47 & 0.011 & $0.3(0.1-0.8)$ \\
Age class & Female & 70 & 0.011 & $3.3(1.2-9.0)$ \\
& 29 y/o & 29 & 1.000 & $0.9(0.3-2.3)$ \\
& $30-39 \mathrm{y} / \mathrm{o}$ & 44 & 0.080 & $0.4(0.2-1.1)$ \\
& $40-49 \mathrm{y} / \mathrm{o}$ & 42 & 0.015 & $2.8(1.2-6.6)$ \\
Occupation & $50 \mathrm{y} / \mathrm{o}$ & 12 & 0.733 & $0.7(0.1-3.2)$ \\
& Medical doctors & 16 & 1.000 & $0.8(0.2-2.9)$ \\
& Nurses & 33 & 0.008 & $3.2(1.3-7.7)$ \\
& Other co-medicals & 36 & 0.076 & $2.2(0.9-5.2)$ \\
& Relatives of patients & 42 & $<0.001$ & $<0.1(0.0-0.4)$ \\
\hline * Two-tailed. & & & &
\end{tabular}

$\dagger$ Odds ratio of being infected while taking specific precautions. have no persuasive explanation for this apart from occupation: $61.9 \%$ of this stratum was medical doctors or nurses. Considering that nurses were more effectively protected from transmission by the use of masks, the control measures taken by them within $\mathrm{HFH}$ from early in the epidemic were admirable. The lowest frequency of infection was seen in relatives of patients, showing that our study included many relatives who remained uninfected but were nevertheless believed to have had contact. Because nonmatched case-control designs such as this are vulnerable to selection bias, we obtained estimates of the protective effect of masks by means of multivariate logistic regression analysis which entered all other variables significantly associated with infection in univariate analysis. After adjustment for internal confounding variables, the estimated reproduction number was given as 0.7 in Stages 2 and 3. Previous studies have shown that the (effective) reproduction number, defined as the average number of secondary cases generated by one index case in a susceptible population under certain restrictions and interventions, decreases with increasing awareness of the epidemic combined with several public health measures. ${ }^{36,37}$ Using reasonable estimation procedures, another study showed that $R$ significantly decreased after a global alert in most affected countries. ${ }^{38}$ The current study showed that the estimated $R$ decreased below unity after notification of a hospital outbreak, although the estimates were obtained using rough assumptions and the process of estimation was biased by various factors.

In $\mathrm{HFH}$, the rapid increase in awareness, which led to not only strengthened precautionary measures and isolation but also quarantining of health care workers, seems to have been the greatest contributor to successful containment. One reason for this quick response could be attributed to the background of secondary cases that arose mainly from health care workers who had close contact with the index case. Almost all staff members working or on duty in the earliest days of the 
TABLE 5

Interactions between wearing masks and other variables on the infection

\begin{tabular}{|c|c|c|c|c|c|c|}
\hline & \multicolumn{3}{|c|}{ In stage 1} & \multicolumn{3}{|c|}{ In stages 2 and 3} \\
\hline & $\begin{array}{l}\text { Odds for } \\
\text { masks }(+)\end{array}$ & $\begin{array}{l}\text { Odds for } \\
\text { masks }(-)\end{array}$ & $\begin{array}{l}\text { Odds } \\
\text { ratio* }\end{array}$ & $\begin{array}{l}\text { Odds for } \\
\text { masks }(+)\end{array}$ & $\begin{array}{l}\text { Odds for } \\
\text { masks (-) }\end{array}$ & $\begin{array}{l}\text { Odds } \\
\text { ratio* }\end{array}$ \\
\hline \multicolumn{7}{|l|}{ Gowns } \\
\hline$(+)$ & 0.3 & 0.6 & 0.5 & $<0.1$ & 2.0 & 0.2 \\
\hline$(-)$ & 0.3 & 0.5 & 0.6 & $\mathrm{NC}$ & $\mathrm{NC}$ & $\mathrm{NC}$ \\
\hline \multicolumn{7}{|l|}{ Sex } \\
\hline (male) & 0.1 & 0.2 & 1.0 & 0.0 & 0.0 & $\mathrm{NC}$ \\
\hline (female) & 0.2 & 0.8 & 0.2 & 0.1 & $\mathrm{NC}$ & $\mathrm{NC}$ \\
\hline \multicolumn{7}{|l|}{ Age class } \\
\hline $29 \mathrm{y} / \mathrm{o}$ & 0.1 & 0.4 & 0.3 & 0.0 & $\mathrm{NC}$ & $\mathrm{NC}$ \\
\hline $30-39$ y/o & 0.1 & 0.3 & 0.5 & 0.0 & $\mathrm{NC}$ & $\mathrm{NC}$ \\
\hline $40-49 \mathrm{y} / \mathrm{o}$ & 0.3 & 0.8 & 0.3 & 0.1 & 1.0 & 0.1 \\
\hline $50 \mathrm{y} / \mathrm{o}$ & 0.2 & 0.2 & 1.0 & 0.0 & $\mathrm{NC}$ & $\mathrm{NC}$ \\
\hline \multicolumn{7}{|l|}{ Occupation } \\
\hline (Medical doctors) & $\mathrm{NC}$ & 0.6 & $\mathrm{NC}$ & 0.0 & 0.0 & $\mathrm{NC}$ \\
\hline (Nurses) & 0.2 & 1.6 & 0.1 & 0.1 & 0.0 & $\mathrm{NC}$ \\
\hline (Other co-medicals) & 0.5 & 0.5 & 1.2 & & & \\
\hline (Relatives of patients) & $\mathrm{NC}$ & 0.1 & $\mathrm{NC}$ & & & \\
\hline
\end{tabular}

$\mathrm{NC}=$ not calculable

* Odds ratio of being infected while taking specific precautions.

outbreak (in Stage 1) were severely infected. ${ }^{39,40}$ Another reason might be due to the efforts led mainly by Dr. Carlo Urbani, who suggested quick improvements in the precautionary measures taken and isolation. ${ }^{29}$ As a result, transmission leakage into the community was prevented, thus having a huge impact on the chains of transmission. ${ }^{24}$ In HFH, those who were exposed implemented precautionary and other controlling measures quickly and efficiently, and the epidemic consequently declined to extinction.

In the interests of objective interpretation, the limitations of our study design must be addressed, as follows:

1) A study such as ours in which exposure has a strong intuitive causal link with outcome (i.e., mask usage) is vulnerable to recall bias. Even though we limited our subjects in Stages 2 and 3 to medical doctors and nurses, and cases were appropriately selected according to the probable date of infection and incubation period, our estimates are likely less accurate than would be obtained by blinded or matched case-control study. In addition to this directional bias, further bias may have been introduced by random misclassification, as our records were completed 1 year after the outbreak, and it is therefore possible that some of the precautions were uncertain exposures. The frequent use of masks among controls may have reduced the strength of the associations.

2) Model-generated results must be interpreted cautiously. Although the simulations shown here included only the effect of masks and were considered according to the results of multivariate logistic regression adjusted for internal factors, unknown external confounding factors likely exist. For example, in Stages 2 and 3, although multivariate logistic regression was performed with other variables, the $P$ value obtained was 0.955 , and overall the model was weak. Owing to the scarcity of case records, stratification in this stage failed to separate the effects of masks. Thus, the estimates of the protective effect of masks and reproduction number in this stage may include the effects of other concomitant changes, such as the reduced frequency of contacts and quarantine.
3) There are limitations concerning the simplicity of our model; for example, we neglected the possible differential susceptibility of humans to asymptomatic infections, ${ }^{41,42}$ individual variance in severity and/or prognosis, ${ }^{23,43,44}$ and the highly heterogenous transmission of SARS., ${ }^{4,45}$ Theoretical exercises never replace reality.

4) Finally, because our model was based on a case-control study, the estimates of coverage were biased; principally, coverage in a case-control design is taken from a nonrepresentative sample. Although this study was conducted as a first attempt to incorporate the effect of behavioral factors, which change time-dependently, to model building strategies for the control of directly transmitted airborne diseases, further studies incorporating a number of methodological improvements are required.

In conclusion, given that early recognition that leads to the implementation of protective behaviors and effective control strategies is crucial in hospitals, ${ }^{46}$ we believe our model provides intuitive results that at least partly satisfy the need to evaluate outbreak trajectories based on individual behaviors.

\section{APPENDIX}

Each simulation starts with one index case and is based on a model constructed as follows:

i) The expected number of people who used protection on each subsequent day was determined by the number of susceptible individuals $(S)$, number of contacts per day (к), proportion of individuals who performed the protective behavior $(p)$, and the protective effect of the precautionary measure $(\beta)$, which were obtained based on our survey. The number of infectious contacts, denoted by the product of the number of susceptible individuals $(S)$ and the mean number of contacts ( $\mathrm{K})$, was divided into two subgroups: one that represents protection due to precautionary behaviors against infection with SARS-CoV (SARS-associated coronavirus) and another that does 
a)

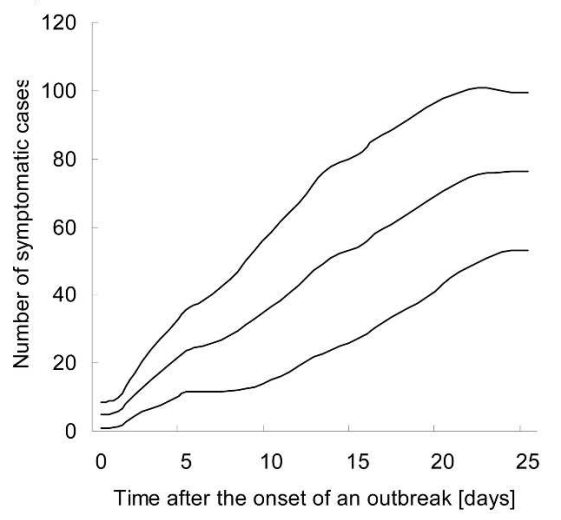

d)

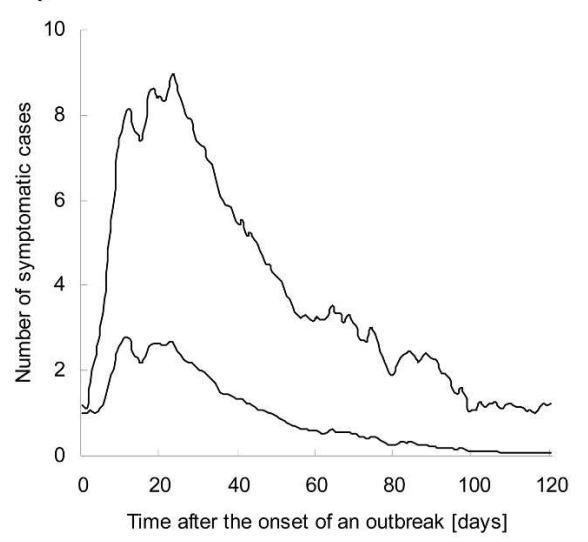

b)

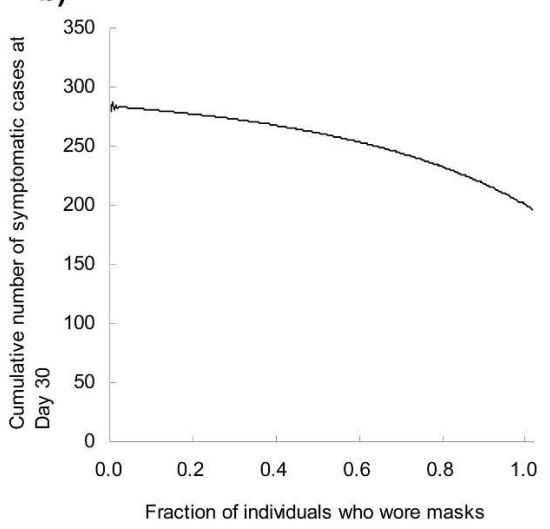

e)

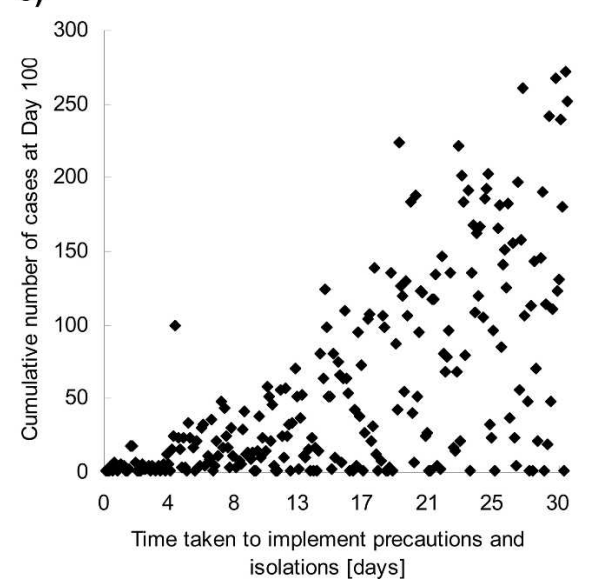

FIGURE 2. Stochastic simulations of a SARS outbreak with dependency on the coverage and protective effect of precautionary behaviors. a, Predicted number of symptomatic cases and corresponding $95 \%$ confidence interval ( $95 \% \mathrm{CI})$ given by 250 simulation runs assuming unchanged (stable) protective behaviors for the entire period. The reproduction number $(R)$ was $4.1 \pm 1.1$. b. Sensitivity of the cumulative number of cases at Day 30 to the coverage of masks. The obtained line represents the simulation based on mean field (without assuming random function with binomial distribution in each transition probability). The protective effect of wearing a mask was fixed $(\beta=0.6)$. c and $\mathbf{d}$, Stochastic simulations of a SARS outbreak with dependency on a combination of precautionary measures and strict isolation. ce, The mean number of incubating individuals and corresponding $95 \%$ CI from 250 runs with changes in protective behaviors combined with strict isolation (lower $95 \%$ CI is $x$-axis). At Day 7, the effectiveness/coverage of precautionary measures used improved from $0.6 / 52.0$ to $0.9 / 89.2$, respectively. At Day 13 , the number of susceptible individuals decreased from 300 to 20 . The reproduction number decreased from $4.1-0.7 \pm 1.1-0.8$. d, The mean $\pm 95 \%$ CI of symptomatic cases given by 250 runs assuming changes in protective behaviors combined with strict isolation. The conditions were the same as those in c. e, Sensitivity of the size of an outbreak (represented by the cumulative number of cases) to the time taken to enhance precautionary measures and implement strict isolation; the combined measures are started at the same time and under the same conditions as in $\mathbf{c}$.

not, according to $(1-p \beta)$. However, these groups were not permanently fixed. The mean of the number of contacts based on our survey was approximated by:

$$
\kappa=\kappa_{1} \pi_{1}+\kappa_{2} \pi_{2}=\kappa_{1} \pi_{1}+\kappa_{1} \ln \left(\mathrm{OR}_{\text {close }}\right) \pi_{2}
$$

where $\kappa_{1}, \kappa_{2}, \pi_{1}$, and $\pi_{2}$ denote the number of casual and close contacts and the fraction of individuals who had casual and close contacts, respectively, while the odds ratio of getting infected with close contact is represented by $\mathrm{OR}_{\text {close }}$ and $N$, respectively.

ii) Both the incubation $(E)$ and symptomatic $(I)$ periods were assumed to be independently and identically distributed following an approximated probability density function with gamma distributions ${ }^{33}$ (denoted by $\gamma_{k}$ and $c_{l}$ for the discretized stages [days] $k$ and $l$, respectively). We divided the probability density functions into $k(i=14)$ and $l(j=12)$ stages; the methodology of approximation by date was previously reported. ${ }^{24}$ The relative measure of infectiousness for the incubation $(E)$ period $(q)$ was assumed to be $0.1 .^{12}$

iii) Based on realistic settings in Vietnam, it was assumed that all individuals were isolated with the onset of early signs of clinical symptoms under the isolation measures; and for simplicity, the effect of quarantine was neglected. When considering strict isolation, the number of susceptible individuals having contact with SARS patients was limited to 20 (which is the approximate number of ward workers); the number of susceptible individuals was treated as being stable (always $S=20$ ) so that $S$ would not be exhausted thereafter; without isolation there were assumed to be 300 susceptible individuals (which is roughly the total number of people involved in possible contacts in HFH). $N=S+E+I+R$, and background mortality was neglected. The resulting simplest difference equations were formulated as follows: 


$$
\begin{aligned}
S(t+1) & =\exp \left[-\kappa(1-p \beta) \frac{I+q E}{N}\right] S(t) \\
E_{1}(t+1) & =\left\{1-\exp \left[-\kappa(1-p \beta) \frac{I+q E}{N}\right]\right\} S(t) \\
E_{k}(t+1) & =\left(1-\gamma_{k-1}\right) E_{k-1}(t) \\
I_{1}(t+1) & =\sum_{k=1}^{i} \gamma_{k} E_{k}(t) \\
I_{l}(t+1) & =\left(1-c_{l-1}\right) I_{l-1}(t) \\
R(t+1) & =R(t)+\sum_{l=1}^{j} c_{l} I_{l}(t)
\end{aligned}
$$

Based on the forward stepwise logistic regression result in the case-control study, and to facilitate understanding, $p$ and $\beta$ were used only to represent the use of masks. However, the protective effect, $\beta$, was obtained from the result of further multiple logistic regression which entered all other significantly associated variables (in univariate analysis). All terms shown here as products of a probability and a state variable were generated in our simulations by using random variables with binomial distributions. Under these assumptions and using mean length of incubation and symptomatic periods, the reproduction number $(R)$ is given by:

$$
R=\kappa(1-p \beta)\left(\frac{q}{\gamma}+\frac{1}{c}\right)
$$

where $\gamma^{-1}$ and $c^{-1}$ are the means of the incubation and symptomatic periods in days, respectively. The basic reproduction number was estimated by

$$
R_{0}=\frac{R}{(1-p \beta)}
$$

For the purpose of mathematical convenience, although unrealistic, our model assumed homogenous mixing as well as all infectious individuals being equally infectious.

Received August 3, 2004. Accepted for publication January 19, 2005.

Acknowledgments: H.N. would like to pay his respects to Dr. Carlo Urbani, an Italian epidemiologist who worked for the WHO Office in Hanoi, whose great achievements in controlling SARS in HFH inspired this study. The authors are grateful to Profs. Masayuki Kakehashi (Hiroshima University, Japan), Minato Nakazawa (Gunma University, Japan), and Klaus Dietz (University of Tubingen, Germany) for their helpful comments and discussions. The authors would also like to thank Dr. Nguyen Le Hang, Ms. Pham Thi Phuong Thuy, and Ms. Nguyen Thi Thu Ha for their help in our survey. H.N. is also grateful to the Japanese Foundation for AIDS Prevention for supporting his stay in the United Kingdom.

Financial support: The study was partly supported by a Health and Labor Sciences Research Grant for "Special Research" entitled "Research on the nosocomial transmission of SARS" awarded by the Ministry of Health, Labor and Welfare, Japan.

Authors' addresses: Hiroshi Nishiura and Roy M. Anderson, Department of Infectious Disease Epidemiology, Faculty of Medicine, Imperial College London, Norfolk Place, London, W2 1PG, United Kingdom. Tadatoshi Kuratsuji, Naoto Keicho, Teruo Kirikae, and Takehiko Sasazuki, Research Institute, International Medical Center of Japan, Toyama 1-21-1, Shinjuku-ku, Tokyo, 162-8655, Japan. Tran Quy and Nguyen Chi Phi, Bach Mai Hospital, Giai Phong Street, Hanoi, Vietnam. Vo Van Ban, Hanoi French Hospital, 1 Phuong Mai Street, Dong Da, Hanoi, Vietnam. Le Dang Ha, National Institute for Clinical Research in Tropical Medicine, Bach Mai Hospital, Giai Phong Street, Hanoi, Vietnam. Hoang Thuy Long, National Institute of Hygiene and Epidemiology, 1 Yersin Street, Hanoi, Vietnam. Hiroshi Nishiura and Hideki Yanai, The Research Institute of Tuberculosis, Japan Anti-Tuberculosis Association, Matsuyama 3-1-24, Kiyose-shi, Tokyo, 204-8533, Japan.

Reprint requests: Tadatoshi Kuratsuji, Research Institute, International Medical Center of Japan, 1-21-1, Toyama Shinjuku-ku, Tokyo, 162-8655, Japan, Telephone: 81-3-3202-7181, Fax: 81-3-5273-4526, Email: kuratsuji@ri.imcj.go.jp.

\section{REFERENCES}

1. Communicable Disease Surveillance \& Response (CDSR), Update 96-Taiwan, China: SARS transmission interrupted in last outbreak area. Geneva, Switzerland: World Health Organization (WHO), 5 July, 2003. Available at http://www.who.int/csr/ don/2003_07_05/en/.

2. Communicable Disease Surveillance \& Response (CDSR), China confirms SARS infection in another previously reported case; summary of cases to date-Update 5. Geneva, Switzerland: World Health Organization (WHO), 30 April, 2004. Available at http://www.who.int/csr/don/2004_04_30/en/.

3. Yu IT, Li TW, Wong TW, Tam W, Chan AT, Lee JHW, Leung DYC, Ho T, 2004. Evidence of airborne transmission of the severe acute respiratory syndrome virus. $N$ Engl J Med 350: 1731-1739.

4. Shen Z, Ning F, Zhou W, He X, Lin C, Chin DP, Zhu Z, Schunchat A, 2004. Superspreading SARS events, Beijing, 2003. Emerg Infect Dis 10: 256-260.

5. Centers for Disease Control and Prevention, 2003. Severe acute respiratory syndrome-Singapore, 2003. MMWR Morb Mortal Wkly Rep 52: 405-411.

6. Tsang KW, Ho PL, Ooi GC, Yee WK, Wang T, Chan-Yeung M, Lam WK, Set WH, Yam LY, Cheung TM, Wong PC, Lam B, Ip MS, Chan J, Yuen KY, Lai KN, 2003. A cluster of cases of severe acute respiratory syndrome in Hong Kong. $N$ Engl $J$ Med 348: 1977-1985.

7. Varia M, Wilson S, Sarwal S, McGeer A, Gournis E, Galanis E, Henry B, 2003. Investigation of a nosocomial outbreak of severe acute respiratory syndrome (SARS) in Toronto, Canada. Can Med Assoc J 169: 285-292.

8. Tsang T, Lam TH, 2003. SARS: public health measures in Hong Kong. Respirology 8: S46-S48.

9. Pang X, Zhu Z, Xu F, Guo J, Gong X, Liu D, Liu Z, Chin DP, Feikin DR, 2003. Evaluation of control measures implemented in the severe acute respiratory syndrome outbreak in Beijing, 2003. J Am Med Assoc 290: 3215-3221.

10. Ho W, 2003. Guideline on management of severe acute respiratory syndrome (SARS). Lancet 361: 1313-1315.

11. Abdullah ASM, Tomlinson B, Cockram CS, Thomas GN, 2003. Lessons from the severe acute respiratory syndrome outbreak in Hong Kong. Emerg Infect Dis 9: 1042-1045.

12. Chowell G, Fenimore PW, Castillo-Garsow MA, Castillo-Chavez C, 2003. SARS outbreaks in Ontario, Hong Kong and Singapore: the role of diagnosis and isolation as a control mechanism. J Theor Biol 224: 1-8.

13. Ho PL, Tang XP, Seto WH, 2003. SARS: hospital infection control and admission strategies. Respirology 8: S41-S45.

14. Lee N, Sung JJ, 2003. Nosocomial transmission of SARS. Curr Infect Dis Rep 5: 473-476.

15. Dwosh HA, Hong HH, Austgarden D, Herman S, Schabas R, 2003. Identification and containment of an outbreak of SARS in a community hospital. Can Med Assoc J 168: 1415-1420.

16. Lee N, Hui D, Wu A, Chan P, Cameron P, Joynt GM, Ahuja A, Yung MY, Leung CB, To KF, Lui SF, Szeto CC, Chung S, Sung JJY, 2003. A major outbreak of severe acute respiratory syndrome in Hong Kong. N Engl J Med 348: 1986-1994.

17. Chow KY, Lee CE, Ling ML, Heng DMK, Yap SG, 2004. Outbreak of severe acute respiratory syndrome in a tertiary hospital in Singapore, linked to an index patient with atypical presentation: epidemiological study. Br Med J 328: 195 [doi: 10.1136/bmj.37939.465729.44].

18. Seto WH, Tsang D, Yung RWH, Ching TY, Ng TK, Ho M, Ho LM, Peiris JSM, and Advisors of Expert SARS Group of Hospital Authority, 2003. Effectiveness of precautions against droplets and contact in prevention of nosocomial transmission 
of severe acute respiratory syndrome (SARS). Lancet 361: $1519-1520$.

19. Nishiura H, Patanarapelert K, Sriprom M, Sarakorn W, Sriyab S, Tang IM, 2004. Modelling potential responses to severe acute respiratory syndrome in Japan: the role of initial attack size, precaution, and quarantine. J Epidemiol Community Health 58: 186-191.

20. Ho AS, Sung JJY, Chan-Yeung M, 2003. An outbreak of severe acute respiratory syndrome among hospital workers in community hospital in Hong Kong. Ann Intern Med 139: 564-567.

21. Lau JTF, Fung KS, Wong TW, Kim JH, Wong E, Chung S, Ho D, Chan LY, Lui SF, Cheng A, 2004. SARS transmission among hospital workers in Hong Kong. Emerg Infect Dis 10: 280-286.

22. Yin WW, Gao LD, Lin WS, Gao LD, Lin WS, Du L, Zhang XC, Zou Q, Li LH, Liang WJ, Peng GW, He JF, Yu DW, Zhou DH, Lin JY, Zeng G, 2004. Effectiveness of personal protective measures in prevention of nosocomial transmission of severe acute respiratory syndrome. Zhonghua Liu Xing Bing Xue Za Zhi 25: 18-22.

23. Vu HT, Leitmeyer KC, Le DH, Miller MJ, Nguyen QH, Uyeki TM, Reynolds MG, Aagesen J, Nicholson KG, Vu QH, Bach HA, Plant AJ, 2004. Clinical description of a completed outbreak of SARS in Vietnam, February-May 2003. Emerg Infect Dis 10: 334-338.

24. Lloyd-Smith JO, Galvani AP, Getz WM, 2003. Curtaining transmission of severe acute respiratory syndrome within a community and its hospital. Proc R Soc Lond B 270: 1979-1989.

25. Ministry of Health, Vietnam, 2003. Severe acute respiratory syndrome (SARS): Epidemiology, clinical aspects, treatment, prevention and control. Hanoi, Vietnam: Medical Publishing House.

26. Vu TH, Cabau JF, Nguyen NT, Lenoir M, 2003. SARS in Northern Vietnam. N Engl J Med 348: 2035.

27. Fleury M, Rathat C, An K, Vu TH, Nguyen NT, Manuguerra JC, 2003. Epidemiology of SARS: mission of the emergency medical department of the French Hospital of Hanoi. Med Trop 63: 287-290.

28. Le DH, Bloom SA, Nguyen QH, Maloney SA, Mai LQ, Leitmeyer KC, Anh BH, Reynolds MG, Montgomery JM, Comer JA, Horby PW, Plant AJ, 2004. Lack of SARS transmission among public hospital workers, Vietnam. Emerg Infect Dis 10: 265-268.

29. Reilley B, Herp MV, Sermand D, Dentico N, 2004. SARS and Carlo Urbani. $N$ Engl J Med 348: 1951-1952.

30. Centers for Disease Control and Prevention, Severe Acute Respiratory Syndrome (SARS): Frequently asked questions about SARS. Atlanta, GA: Centers for Disease Control and Prevention, 2003. Available at: http://www.cdc.gov/ncidod/ sars/faq.htm.

31. Development R, Core team, 2004. R; a language and environment for statistical computing. Vienna: R Foundation for Statistical Computing. Available at www.r-project.org.

32. Lipsitch M, Cohen T, Cooper B, Robins JM, Ma S, James L, Gopalakrishna G, Chew SK, Tan CC, Samore MH, Fisman D, Murray M, 2003. Transmission dynamics and control of severe acute respiratory syndrome. Science 300: 1966-1970.

33. Donnelly CA, Ghani AC, Leung GM, Hedley AJ, Fraser C, Riley S, Abu-Raddad LJ, Ho LM, Thach TQ, Chau P, Chan KP, Lam TH, Tse LY, Tsang T, Liu SH, Kong JHB, Lau EMC,
Ferguson NM, Anderson RM, 2003. Epidemiological determinants of spread of causal agent of severe acute respiratory syndrome in Hong Kong. Lancet 361: 1761-1766.

34. Centers for Disease Control and Prevention, 2003. Cluster of severe acute respiratory syndrome cases among protected health-care workers - Toronto, Canada. MMWR Morb Mortal Wkly Report 52: 433-436.

35. Leung TF, Ng PC, Cheng FWT, Lyon DJ, So KW, Hon EKL, Li AM, Li CK, Wong GWK, Nelson EAS, Hui J, Sung RYT, Yam MC, Fok TF, 2004. Infection control for SARS in a tertiary paediatric centre in Hong Kong. J Hosp Infect 56: 215222.

36. Riley S, Fraser C, Donnelly CA, Ghani AC, Abu-Raddad LJ, Hedley AJ, Leung GM, Ho LM, Lam TH, Thach TQ, Chau P, Chan KP, Lo SV, Leung PY, Tsang T, Ho W, Lee KH, Lau EMC, Ferguson NM, Anderson RM, 2003. Transmission dynamics of the etiological agent of SARS in Hong Kong: impact of public health interventions. Science 300: 1961-1966.

37. Hsieh YH, Lee JY, Chang HL, 2004. SARS epidemiology modeling. Emerg Infect Dis 10: 1165-1167.

38. Wallinga J, Teunis P, 2004. Different epidemic curves for severe acute respiratory syndrome reveal similar impacts of control measures. Am J Epidemiol 15: 509-516.

39. Ban VV, 2003. The outbreak of SARS in Vietnam French Hospital and countermeasure of control. Seminar on SARS Control. Hanoi, Vietnam: Ministry of Health, Vietnam, 26-34.

40. Long HT, 2003. Conducting and managing activities for SARS control in Vietnam. Seminar on SARS Control. Hanoi, Vietnam: Ministry of Health, Vietnam, 1-4.

41. Guan Y, Zheng BJ, He YQ, Liu XL, Zhuang ZX, Cheung CL, Luo SW, Li PH, Zhang LJ, Guan YJ, Butt KM, Wong KL, Chan KW, Lim W, Shortridge KF, Yuen KY, Peiris JSM, Poon LLM, 2003. Isolation and characterization of viruses related to the SARS coronavirus from animals in Southern China. Science 302: 276-278.

42. Ho KY, Singh KS, Habib AG, Ong BK, Lim TK, Ooi EE, Sil BK, Ling AE, Bai XL, Tambyah PA, 2004. Mild illness associated with severe acute respiratory syndrome coronavirus infection: lessons from a prospective seroepidemiologic study of healthcare workers in a teaching hospital in Singapore. J Infect Dis 189: 642-647.

43. Peiris JS, Chu CM, Cheng VCC, Chan KS, Hung IFN, Poon LLM, Law KI, Tang BSF, Hon TYW, Chan CS, Chan KH, Ng JSC, Zheng BJ, Ng WL, Lai RWM, Guan Y, Yuen KY, 2003. Clinical progression and viral load in a community outbreak of coronavirus-associated SARS pneumonia: a prospective study. Lancet 361: 1767-1772.

44. Chan JW, Ng CK, Chan YH, Mok TY, Lee S, Chu SY, Law WL, Lee MP, Li PC, 2003. Short term outcome and risk factors for adverse clinical outcomes in adults with severe acute respiratory syndrome (SARS). Thorax 58: 686-689.

45. Masuda N, Konno N, Aihara K, 2004. Transmission of severe acute respiratory syndrome in dynamical small-world networks. Phys Rev E Stat Nonlin Soft Matter Phys 69: 031917.

46. Manocha S, Walley KR, Russell JA, 2003. Severe acute respiratory distress syndrome (SARS): a critical care perspective. Crit Care Med 31: 2684-2692. 